\title{
Application of Electrocautery Needle Knife Combined with Balloon Dilatation versus Balloon Dilatation in the Treatment of Tracheal Fibrotic Scar Stenosis
}

\author{
Liyan $\mathrm{Bo}^{\mathrm{a}}$ Congcong $\mathrm{Li}^{\mathrm{b}}$ Min Chen ${ }^{\mathrm{a}}$ Deguang $\mathrm{Mu}^{\mathrm{a}}$ Faguang $\mathrm{Jin}^{\mathrm{a}}$ \\ a Department of Respiratory and Critical Care Medicine, Tangdu Hospital, Fourth Military Medical University, Xi'an, \\ PR China; bepartment of Respiratory and Critical Care Medicine, General Hospital of Shenyang Military Area \\ Command, Shenyang, PR China
}

\section{Keywords}

Electrocautery needle knife $\cdot$ Balloon dilatation $\cdot$ Tracheal

fibrotic stenosis · Intubation · Tracheotomy

\begin{abstract}
Background: Electrocautery needle knives can largely reduce scar and granulation tissue hyperplasia and play an important role in treating patients with benign stricture. Objective: The aim of this retrospective study was to evaluate the efficacy and safety of electrocautery needle knife combined with balloon dilatation versus balloon dilatation alone in the treatment of tracheal stenosis caused by tracheal intubation or tracheotomy. Methods: We retrospectively analysed the clinical data of 43 patients with tracheal stenosis caused by tracheotomy or tracheal intubation in our department from January 2013 to January 2016. Among these 43 patients, 23 had simple web-like stenosis and 20 had complex stenosis. All patients were treated under general anaesthesia, and the treatment methods were (1) balloon dilatation alone, (2) needle knife excision of fibrotic tissue combined with balloon dilatation, and (3) needle knife radial incision of fibrotic tissue combined with balloon dilatation. Results: After treatment the symptoms, such as shortness of breath,
\end{abstract}

were markedly improved immediately in all cases. The stenosis degree of patients who were treated with the electrocautery needle knife combined with balloon dilatation had better improvement compared with that of those treated with balloon dilatation treatment alone after 3 months $(0.45 \pm 0.04$ vs. $0.67 \pm 0.05, p<0.01)$, and the proportion of restenosis occurrence that required further treatment was decreased at 6 months (46.9 vs. $81.8 \%$ ), especially for the web-like stenosis patients, as most of their stenoses dilated with no obvious restenosis and achieved clinical cure. Conclusion: Electrocautery needle knife combined with balloon dilatation is an effective and safe treatment for tracheal fibrotic stenosis compared with balloon dilatation alone.

(c) 2017 S. Karger AG, Basel

\section{Introduction}

Airway stenosis includes benign airway stenosis and malignant airway stenosis. Benign central airway stenosis is a common group of diseases of the respiratory system,

L. Bo and C. Li contributed equally to this article.

\section{KARGER}

(c) 2017 S. Karger AG, Basel

E-Mail karger@karger.com

www.karger.com/res
Faguang Jin and Deguang $\mathrm{Mu}$

Department of Respiratory and Critical Care Medicine

Tangdu Hospital, Fourth Military Medical University

Xinsi Road 1, Xi'an 710038 (PR China)

E-Mail jinfag@ fmmu.edu.cn or deguang@fmmu.edu.cn 

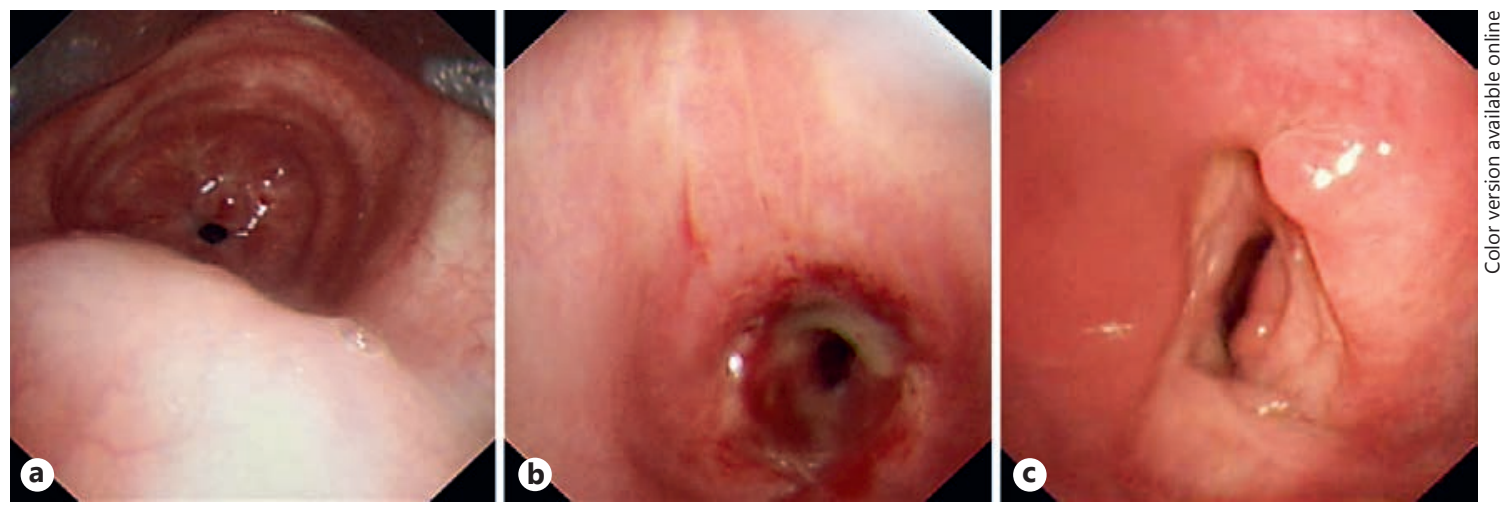

Fig. 1. Tracheal stenosis types. a Simple web-like stenosis. b Bottleneck stenosis. c Mixed constriction.

and it is mainly caused by bronchial tuberculosis, endotracheal intubation, tracheotomy, surgery, foreign body, benign tumour, tracheal amyloidosis, relapsing polychondritis, or airway wall collapse $[1,2]$. The number of patients complicated by tracheal fibrotic stenosis after tracheotomy or tracheal intubation increases gradually each year. There are few treatment methods for this complication, and surgery has been the first choice. However, due to the high risk of thoracic surgery, the heavy economic burden, or inability to tolerate surgical treatment, the therapy of tracheal fibrotic stenosis after tracheotomy or tracheal intubation is a very difficult and challenging problem. Since the twentieth century, interventional pulmonology technologies have been widely used and have become important choices in the treatment of benign central airway stenosis, and surgery has been partly replaced by interventional bronchoscopic therapy $[3,4]$. Interventional bronchoscopic therapies include highpressure balloon dilatation, argon plasma coagulation, cryotherapy, laser, and stents. In general, the patients' symptoms are relieved rapidly after interventional bronchoscopic therapy [5]. However, the therapies can also lead to airway mucosal injury, stimulate the hyperplasia of scar tissue, cause short-term granulation tissue hyperplasia, and eventually lead to airway stenosis again and maybe even worse symptoms, endangering the lives of patients [6]. Thus, it is important to choose a suitable interventional therapeutic plan for patients with benign central airway stenosis [7]. Here, we mainly evaluated the efficacy of an electrocautery needle knife and balloon dilatation.

Electrocautery needle knives are widely used in surgery because of their shorter operation time, lower bleeding volume, smaller wounds, and other advantages. As the contact area between the needle knife and the airway mucosa is small, the airway damage area is small as well [8]. As a result, the electrocautery needle knife can largely reduce the formation of scar and granulation tissue hyperplasia and might play an important role in treating patients with benign stricture. This article mainly evaluates the role of an electrocautery needle knife combined with balloon dilatation in the treatment of benign tracheobronchial stenosis, especially in patients with complex benign tracheal stenosis.

\section{Subjects and Methods}

\section{Clinical Data}

We retrospectively analysed 11 patients with tracheal stenosis treated with balloon dilatation alone and 32 patients with tracheal stenosis treated with electrocautery needle knife combined with balloon dilatation in our department from January 2013 to January 2016. The inclusion and exclusion criteria were (a) inclusion of patients with tracheal fibrotic stenosis after tracheotomy or tracheal intubation, (b) exclusion of patients with concurrent active tuberculosis or lung tumour, and (c) exclusion of those with other fatal diseases. The 43 cases of tracheal stenosis included 31 males and 12 females; their ages were between 22 and 61 years. Among them, 27 patients had stenosis after tracheal intubation, and the other 16 had stenosis after tracheal intubation and subsequent tracheotomy.

Tracheal stenosis was divided into simple web-like stenosis and complex stenosis. As shown in Figure 1, web-like stenosis was defined as mostly circumferential strictures of the trachea involving a short segment of the mucosa (stenosis length $<1 \mathrm{~cm}$ ). Complex stenosis was strictures of the trachea $>1 \mathrm{~cm}$ long. These were further divided into bottleneck stenosis (stenosis occurred gradually, and there was a relatively longer distance from the normal tissue to the narrowest part) and mixed constriction (with the mixed features of web-like stenosis and bottleneck stenosis).

\section{Materials}

The equipment used included a bronchoscope (BF 260; Olympus, Japan), an ultrathin bronchoscope (BF-P260F; Olympus), an 
Table 1. Clinical characteristics: stenosis type, treatment method, and treatment result

\begin{tabular}{|c|c|c|c|c|c|}
\hline Stenosis type & $\begin{array}{l}\text { Patients, } \\
n\end{array}$ & Treatment method & \multicolumn{3}{|c|}{ Effect } \\
\hline $\begin{array}{l}\text { Simple web-like } \\
\text { tracheal stenosis }\end{array}$ & 18 & $\begin{array}{l}\text { electrocautery needle knife radial incision combined } \\
\text { with balloon dilatation }\end{array}$ & 18 & 0 & 0 \\
\hline \multirow[t]{2}{*}{ Mixed constriction } & 3 & high-pressure balloon dilatation & 0 & 2 & 1 \\
\hline & 6 & $\begin{array}{l}\text { electrocautery needle knife ring/radial incision } \\
\text { combined with balloon dilatation }\end{array}$ & 3 & 3 & 0 \\
\hline
\end{tabular}

electrocautery needle knife (Olympus), a balloon dilatation catheter (Johnson \& Johnson, USA), and a pressure pump (Johnson \& Johnson).

\section{Treatment Methods: Preoperative Preparation}

CT examination and airway reconstruction were performed before treatment to evaluate the degree of stenosis, and the percentage of normal size was recorded. Routine blood test, blood coagulation, arterial blood gas, kidney function, and electrocardiogram examination were also performed before operation. The patients were forbidden to take any anticoagulation or antiplatelet drugs $72 \mathrm{~h}$ before the operation and had to abstain from food and water for $6 \mathrm{~h}$ before the operation.

\section{Treatment Methods: Operation Procedure}

The patients were operated on under general anaesthesia because the electrocautery needle knife is very sharp and might damage the airway when the patients coughs, and even lead to the risk of massive bleeding.

After anaesthesia, ventilator-assisted ventilation was performed though a laryngeal mask or by insertion of a rigid bronchoscope. The patient's stenosis was examined again with a bronchoscope or ultrathin bronchoscope. In the balloon dilatation alone group, the diameter of the balloon was selected based on the diameter of stenosis, and in one cycle of treatment, the dilatation was performed 5 times for $1 \mathrm{~min}$ each. These treatment procedures were performed once a week until the diameter was $>50 \%$ of the normal size and the lesion was stable, with no obvious restenosis at the next week's examination.

In the electrocautery needle knife combined with balloon dilatation group, there were two statuses. For patients with a simple web-like stenosis, we radially cut the narrow part of the fibrotic tissue (normal in four to six directions) and then dilated with a high-pressure balloon. For patients with complex stenosis, if the fibrotic tissue lesion was relatively tough or hard, we circularly resected the fibrotic lesions with the electrocautery needle knife and then performed balloon dilatation. If the texture was soft, we radially incised the scar tissue with the needle knife followed by hyperbaric balloon dilatation. The balloon dilatation treatment was the same in as the balloon dilatation alone group. These treatment procedures were performed once a week until the diameter was $>50 \%$ of the normal size and the lesion was stable, with no obvious restenosis at the next week's examination.

\section{Therapeutic Efficacy Evaluation}

As there were no standard criteria for the evaluation of the treatment efficacy of tracheal stenosis, we divided the treatment results into three levels: (1) clinical cure: the lesion was stable for $>3$ months; (2) effective: the lesion was stable for $>1$ month but $<3$ months; (3) invalid: lesion stability lasted $<1$ month. We also measured the percentage of stenosis area before treatment and 3 months after treatment. For long-term efficacy evaluation, we recorded the proportion of restenosis occurrence that needed further treatment. Data are reported as mean values \pm standard error. Comparisons between groups were made by the unpaired $t$ test, and differences in the mean values were considered to be significant at $p<0.05$.

\section{Results}

The clinical characteristics (including stenosis type, treatment method, and treatment result) of the 43 cases are shown in Table 1. For the patients treated with balloon dilatation alone, the diameter of the balloon was selected based on the diameter of the stenosis, and in one cycle of treatment the dilatation was performed 5 times for $1 \mathrm{~min}$ each. The patients treated with electrocautery needle knife combined with balloon dilatation included 18 cases of simple web-like stenosis and 14 cases of complex stenosis. For these 18 patients with web-like stenosis, after 2-3 applications of the electrocautery needle knife combined with high-pressure balloon dilatation, their shortness of breath symptoms improved significantly and the tracheal stenosis significantly expanded. Their condition was stable within 3 months, and no obvious retraction stenosis appeared 
Table 2. Treatment details and outcomes

\begin{tabular}{lllll}
\hline Treatment method & Patients, $n$ & $\begin{array}{l}\text { Stenosis degree } \\
\text { before treatment }\end{array}$ & $\begin{array}{l}\text { Stenosis degree } \\
\text { after treatment }\end{array}$ & $\begin{array}{l}\text { Treatment } \\
\text { times }\end{array}$ \\
\hline HPBD & 11 & $0.83 \pm 0.02$ & $0.67 \pm 0.05$ & $4.27 \pm 0.38$ \\
ENK+HPBD & 32 & $0.84 \pm 0.01$ & $0.45 \pm 0.04$ & $3.56 \pm 0.22$ \\
\hline$p$ value & & 0.73 & $<0.01$ & 0.11 \\
\hline
\end{tabular}

ENK, electrocautery needle knife; HPBD, high-pressure balloon dilatation.

Fig. 2. a Stenosis degree of the high-pressure balloon dilatation (HPBD) group and the electrocautery needle knife combined with $\mathrm{HPBD}(\mathrm{ENK}+\mathrm{HPBD})$ group before treatment. b Stenosis degree of the HPBD group and the ENK+HPBD group after treatment.

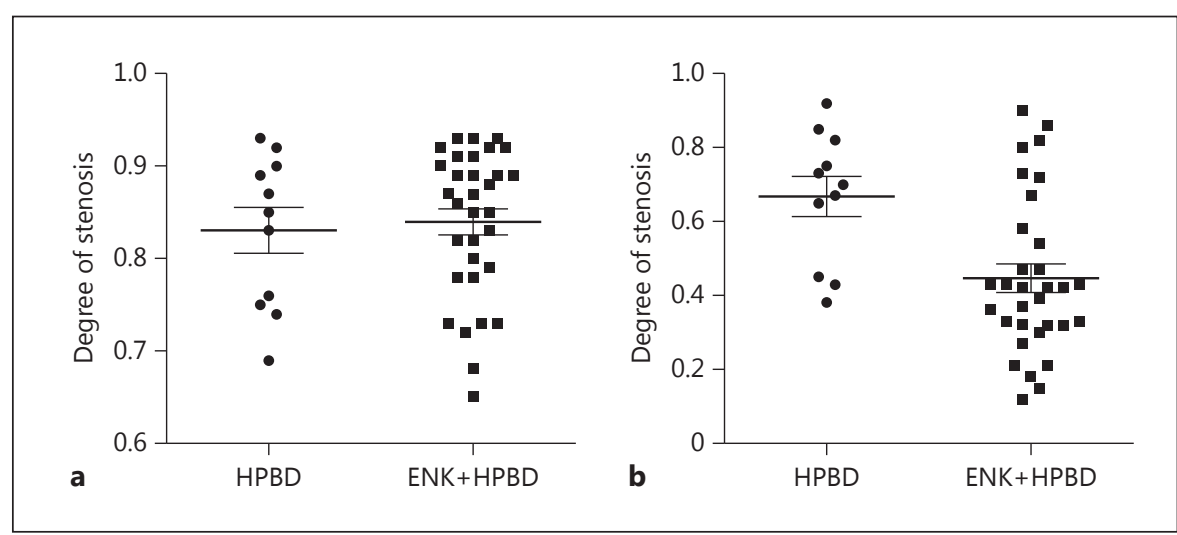

again. For the 14 patients with complex stenosis, 6 had mixed constriction and the other 8 had bottleneck stenosis. Of the 6 mixed constriction cases, the initial electrocautery needle knife ring/radial incision combined with balloon dilatation was applied in 3 cases. After 2-4 rounds of treatment, the stenosis was stable, and there was no significant reduction at 3-month follow-up. The other 3 cases were repeatedly treated with balloon dilatation, argon plasma coagulation, or cryotherapy, and the airway still retracted. However, after performing electrocautery needle knife ring/radial incision combined with balloon dilatation 4-5 times, their conditions became stable. All 8 cases of bottleneck stenosis were treated with circumcision combined with balloon dilatation therapy. Three of them were stable and achieved clinical cure after 4-5 rounds of treatment, the treatment reached the effective level in 1 case, and the other 4 patients were still deteriorating and underwent $\mathrm{T}$ catheter treatment by surgery. Balloon dilatation alone showed poor efficacy in treating complex stenosis.

As shown in Table 2 and Figure 2, there was no difference $(p=0.73)$ in the stenosis degree between the highpressure balloon dilatation group and the electrocautery needle knife combined with balloon dilatation group before treatment. However, the patients treated with the

Application of Electrocautery Needle Knife Combined with Balloon Dilatation electrocautery needle knife combined with balloon dilatation had better improvement compared with the balloon dilatation alone group 3 months after treatment $(0.45 \pm 0.04$ vs. $0.67 \pm 0.05, p<0.01)$ (Fig. 2,3$)$, and the proportion of restenosis occurrence that needed further treatment decreased in the former group at 6 months (46.9 vs. $81.8 \%$ ), especially for the web-like stenosis patients (Fig. 4). Their stenosis dilated with no obvious restenosis and achieved clinical cure.

\section{Complications}

Among the 43 patients, mild mucosal laceration occurred in 5 after dilatation, and the lacerations self-healed without special treatment. There was no subcutaneous emphysema, airway wall perforation, bleeding, or other complication (including endobronchial fires, haemoptysis, haemorrhage, infection, or aspiration pneumonia).

\section{Discussion}

Benign central airway stenosis is an acute, life-threatening disease, and recently the incidence of this disease has increased yearly [9]. The most common type is benign 
Fig. 3. a Change in stenosis degree in the balloon dilatation group. b Change in stenosis degree in the electrocautery needle knife combined with balloon dilatation group.

Fig. 4. Change in stenosis degree in the electrocautery needle knife combined with balloon dilatation group. a Change in stenosis degree in the simple web-like tracheal stenosis patients. b Change in stenosis degree in the complex stenosis patients.
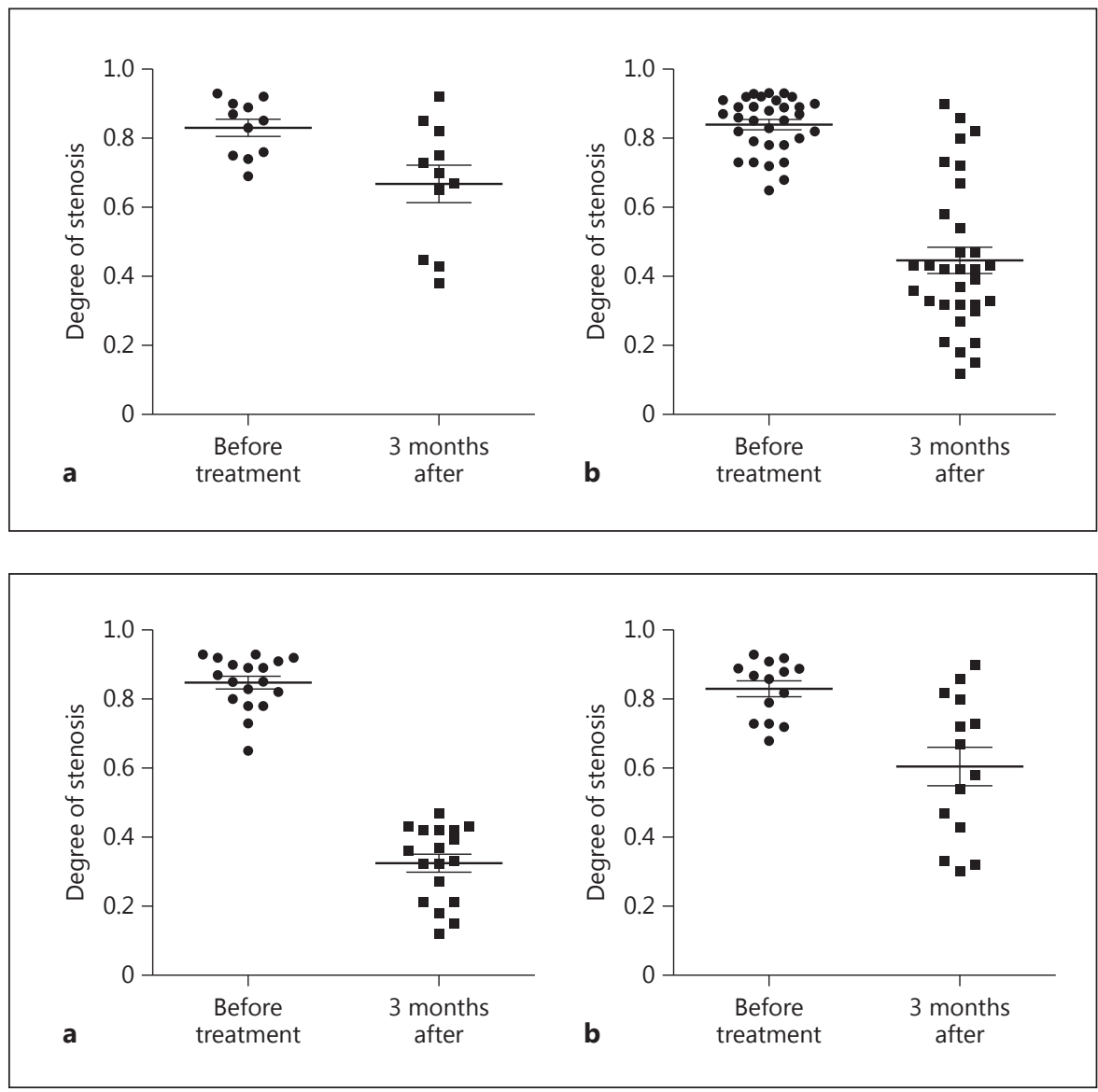

hyperplastic and cicatricial stenosis. With the development of interventional pulmonary methods, lung intervention techniques can quickly open the airway and relieve the symptoms of benign airway stenosis. However, there are still some patients with long-term fibrotic tissue proliferation, leading to restenosis and requiring repeated treatments. The poor efficacy of the treatment aggravates patients' suffering, increases the economic burden, and becomes a vital problem that hinders the use of interventional treatments for benign airway stenosis [10-12]. Therefore, clinicians should be careful in the choice of interventional therapy for benign central airway stenosis. We should choose the appropriate method to treat it according to the specific character of the disease.

The electrocautery needle knife's cutting function comes from the "skin effect" of a high-frequency current. The high voltage and high-frequency current generated on the electrode tip can quickly evaporate the organisation of the surface it contacts, splitting a several-millimetre-deep incision with less bleeding than traditional methods [13]. Here, we reviewed 43 patients with cicatri- cial stricture after tracheotomy or tracheal intubation, including 23 simple web-like stenosis cases and 20 complex tracheal stenosis cases. In the electrocautery needle knife incision combined with balloon dilatation group, there were 32 cases, including 18 simple web-like stenosis cases and 14 complex stenosis cases. Among them, 8 and 3, respectively, had been treated with other methods before, but the outcomes had been ineffective. After we applied needle-knife resection combined with balloon dilatation, the symptoms of these patients improved significantly, tracheal stenosis expanded and did not appear with any obvious retraction, and clinical cure was achieved. These results indicate that needle knife resection combined with balloon dilatation was an effective and maybe a better treatment method for benign tracheal fibrotic stenosis than balloon dilatation alone.

Through the analysis of the treatment procedures and the results of the 43 clinical cases of tracheal stenosis and the related literature, we summarise the following items that need more attention. 
Regarding the indications and treatment methods, radially cutting the narrow part of the fibrotic tissue with an electrocautery needle knife and then dilating with a highpressure balloon is suitable for tough airway scar tissue, especially for patients whose fibrotic lesion is long. When the fibrotic tissue of these patients is tough, balloon dilatation alone is often ineffective. However, if we first cut the fibrotic tissue with the electrocautery needle knife followed by balloon dilatation, the desired effect might be achieved, and, to some extent, the excessive pressure causing tracheal mucosal tears might be avoided [14]. For the patients with complex stenosis, combining a radial cut of the narrow part of the fibrotic tissue with circular resection might be more suitable.

Second, the degree of scar regeneration and the incidence of restenosis were low for the patients treated with electrocautery needle knife compared with argon plasma coagulation or laser ablation, indicating that the treatment effect of electrocautery needle knife with balloon dilatation might be better than that of other methods.

Third, the electrocautery needle knife's cutting is fast and dangerous, and the movement caused by coughing or even breathing may hurt the airway. Thus, general anaesthesia and high-frequency ventilation are required during the operation to eliminate the influence of the patient's respiratory motion.
In summary, improper use of the needle-shaped electric knife might lead to fatal complications, and the technical requirements of the operator are high. However, this technology has good outcomes in treating refractory benign central airway stenosis, especially airway stenosis that is not improved by other methods. Radially or circularly cutting with the needle knife combined with highpressure balloon dilatation can significantly improve the airway mucosa injury severity and fibrous tissue hyperplasia and is worth clinical application and popularisation.

\section{Acknowledgement}

The authors thank the Research Special Fund for Public Welfare Industry of Health (No. 201402024).

\section{Statement of Ethics}

This study was approved by the Regional Ethics Committee of Tangdu Hospital.

\section{Financial Disclosure and Conflicts of Interest}

On behalf of all authors, the corresponding authors state that there is no conflict of interest.

\section{References}

1 Mehta RM, Singla A, Shah A, Loknath C: The "hitch stitch": an effective method of preventing migration in high tracheal stenosis. Respiration 2017;93:106-111.

2 Morales A, Pari M, López-Lisbona R, Cubero N, Dorca J, Rosell A: Colchicine treatment for tracheobronchial amyloidosis. Respiration 2016;91:251-255.

3 Hsu HS, Hsu WH, Huang BS, Huang MH: Surgical treatment of endobronchial tuberculosis. Scand Cardiovasc J 1997;31:79-82.

4 Bolliger CT, Mathur PN, Beamis JF, Becker $\mathrm{HD}$, Cavaliere S, Colt H, Diaz-Jimenez JP, Dumon JF, Edell E, Kovitz KL, Macha HN, Mehta AC, Marel M, Noppen M, Strausz J, Sutedja TG; European Respiratory Society/ American Thoracic Society: ERS/ATS statement on interventional pulmonology. European Respiratory Society/American Thoracic Society. Eur Respir J 2002;19:356-373.
5 Wahidi MM, Herth FJ, Ernst A: State of the art: interventional pulmonology. Chest 2007; 131:261-274.

6 Dutau H, Musani AI, Laroumagne S, Darwiche K, Freitag L, Astoul P: Biodegradable airway stents - bench to bedside: a comprehensive review. Respiration 2015;90:512-521.

7 Gaissert HA, Grillo HC, Wright CD, Donahue DM, Wain JC, Mathisen DJ: Complication of benign tracheobronchial strictures by self-expanding metal stents. J Thorac Cardiovasc Surg 2003;126:744-747.

8 Zhang H, Zhang Z, Li H, Liu D: Discussion on principle and safety of radio knife. Chin Med Equip J 2009;30:105-106.
9 Courey MS: Airway obstruction. The problem and its causes. Otolaryngol Clin North Am 1995;28:673-684.

10 Rahman NA, Fruchter O, Shitrit D, Fox BD Kramer MR: Flexible bronchoscopic management of benign tracheal stenosis: long term follow-up of 115 patients. J Cardiothorac Surg 2010;5:2.

11 Smith ME, Elstad M: Mitomycin C and the endoscopic treatment of laryngotracheal stenosis: are two applications better than one? Laryngoscope 2009;119:272-283.

12 Rafanan AL, Mehta AC: Stenting of the tracheobronchial tree. Radiol Clin North Am 2000;38:395-408.

13 Freitag L: Interventional endoscopic treatment. Lung Cancer 2004;45(suppl 2):S235S238.

14 Mitchell SA: The mechanism of tracheal stenosis. Ear Nose Throat J 1979;58:28-33, 37. 